\title{
Tumor-to-tumor metastasis of diffuse large $B$ cell lymphoma to gastric adenocarcinoma via CXCL12 (SDF-1)/CXCR4 axis: a case report
}

Yusuke Kamihara', Sayaka Murai ${ }^{2}$, Shohei Kikuchi ${ }^{1}$, Akinori Wada ${ }^{1}$, Jun Murakami ${ }^{3}$, Nam H. Dang ${ }^{4}$ and Tsutomu Sato ${ }^{1 *}$

\begin{abstract}
Background: Tumor-to-tumor metastasis is the rare phenomenon in which one tumor exhibits metastatic deposits from another. To the best of our knowledge, there has been no prior reported case of tumor-to-tumor metastasis of a diffuse large $B$ cell lymphoma ( $\mathrm{DLBCL}$ ) to a primary gastric adenocarcinoma.

Case presentation: A 70-year-old man presented with chest discomfort. An echocardiogram showed the presence of a right ventricular tumor. A positron emission tomogram showed multiple foci of abnormal activity in right cervical lymph nodes, cardiac wall, and stomach. A right cervical lymph node biopsy specimen revealed histological features of DLBCL. An esophagogastroduodenoscopy showed a large circumferential ulceration on the gastric body. Subsequent biopsy revealed adenocarcinoma cells surrounded by infiltrating lymphoma cells. On immunohistochemical staining, lymphoma cells were positive for CXCR4 and adenocarcinoma cells were positive for CXCL12/SDF-1. The patient was treated with six cycles of R-CHOP chemotherapy regimen, resulting in a complete remission.
\end{abstract}

Conclusions: This patient's case implies that the interaction between a chemokine and its receptor may be the underlying mechanism for the observed tumor-to-tumor metastasis. Specifically, our case would suggest an involvement of the CXCL12 (SDF-1)/CXCR4 axis in the observed metastasis of DLBCL to primary gastric adenocarcinoma.

Keywords: Tumor-to-tumor metastasis, Gastric adenocarcinoma, CXCL12 (SDF-1)/CXCR4 axis, Case report

\section{Background}

Tumor-to-tumor metastasis is the rare phenomenon in which one tumor is involved by metastatic deposits from another [1]. The recipient tumors are either malignant or benign, with renal cell carcinoma being the most common of the malignant recipients and meningioma being the most common for benign recipients [1], whereas lung and breast cancers are common donors [2]. In this report, a diffuse large B cell lymphoma (DLBCL) metastasizing to a gastric adenocarcinoma is described. No similar

\footnotetext{
*Correspondence: tsutomus@med.u-toyama.ac.jp

1 Department of Hematology, Toyama University Hospital, 2630 Sugitani,

Toyama 930-0194, Japan

Full list of author information is available at the end of the article
}

cases have been found in the accessible literature. Further, as a possible mechanism of tumor-to-tumor metastasis, the involvement of both C-X-C motif chemokine ligand 12/stromal cell-derived factor-1 (CXCL12/SDF1 ) and $\mathrm{C}-\mathrm{X}-\mathrm{C}$ motif chemokine receptor 4 (CXCR4) is examined.

\section{Case presentation}

A 70-year-old man presented to his primary care physician with chest discomfort, palpitation and dizziness. $\mathrm{He}$ denied chest pain, shortness of breath and fatigue. When electrocardiogram revealed ST-segment elevation in all the anterior leads (V1 to V6), the patient was referred to our hospital. An echocardiogram showed 
the presence of a large immobile mass attached to his right ventricle extending to the outflow tract (Fig. 1a). The serum level of soluble interleukin-2 receptor was markedly increased to $6,500 \mathrm{U} / \mathrm{mL}$ (reference range, 122-496 U/mL). ${ }^{18}$ F-Fluorodeoxyglucose (FDG) positron emission tomogram showed multiple foci of abnormal FDG accumulation in right cervical and mediastinal lymph nodes, cardiac wall, and stomach with elevated maximum standardized uptake values of more than 27 (Fig. 1b). A right cervical lymph node biopsy specimen was obtained and revealed histological features of DLBCL, not otherwise specified, with the non-germinal center B-cell-like (non-GCB) immunophenotype, being positive for CD20, BCL6, and MUM1 and negative for CD10. An esophagogastroduodenoscopy (EGD) showed a large circumferential ulceration on the greater curvature of the gastric body (Fig. 2a). Histopathological assessment of the biopsied gastric tumor from nine different sites (white arrows in Fig. 2a) revealed diffuse infiltration of abnormal large lymphoid cells. These cells were positive for CD20, BCL-6 and MUM1 and negative for $\mathrm{CD} 10$. On the basis of these results, the gastric lesion was also determined to be DLBCL, non-GCB subtype. There were no pathological findings suggesting mucosa

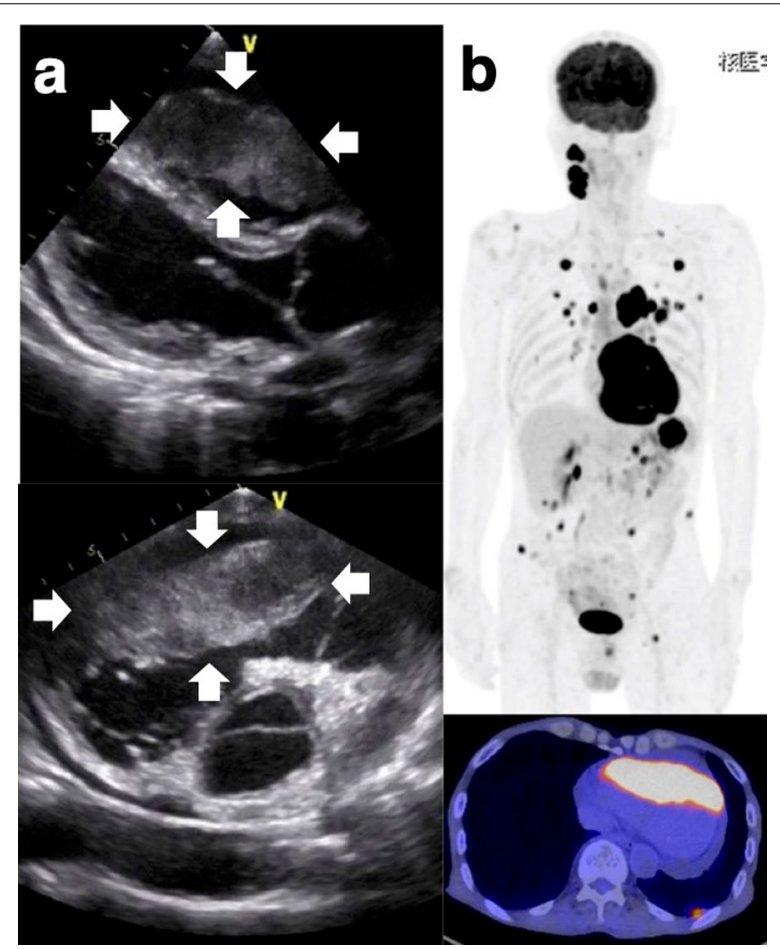

Fig. 1 Imaging findings. a An echocardiogram showed the presence of a large immobile mass attached to the patient's right ventricle extending to the outflow tract. $\mathbf{b}$ An FDG-PET showed multiple foci of abnormal FDG accumulation in right cervical and mediastinal lymph nodes, cardiac wall, and stomach associated lymphoid tissue (MALT) lymphoma such as lymphoepithelial lesions or eosinophilic degeneration of epithelial cells. Helicobacter pylori (H. pylori) was not detected in the biopsy specimens. On the other hand, biopsy specimens from three other sites (red arrows in Fig. 2a) were found to have microtubular glands and cribriform proliferation, suggesting the existence of a moderately differentiated tubular adenocarcinoma (tub2). These adenocarcinoma cells were surrounded by DLBCL cells (Fig. 2b: low-power field, Fig. 2c: high-power field). On immunohistochemical staining using anti-human CXCR4 mouse monoclonal antibody (clone 44716) and anti-human/mouse CXCL12/SDF-1 mouse monoclonal antibody (clone 79018), DLBCL cells were positive for CXCR4 (Fig. 2d) and adenocarcinoma cells were positive for CXCL12/SDF-1 (Fig. 2e). The patient was started on R-CHOP chemotherapy regimen (rituximab, cyclophosphamide, doxorubicin, vincristine, and prednisolone), resulting in a complete remission after six cycles. Follow-up EGD after chemotherapy detected a scar lesion instead of a tumor (Fig. 3). Biopsy specimens from the scar were negative not only for lymphoma cells but also adenocarcinoma cells.

\section{Discussion and conclusion}

The occurrence of both primary gastric lymphoma and gastric adenocarcinoma in the same patient is a rare entity. 56 cases of synchronous occurrence of both primary gastric lymphoma and gastric adenocarcinoma have been summarized by Hamaloglu et al. [3]. In their 56 cases, the majority of the lymphoma cases was MALT (69.6\%) and the association of $H$. pylori with MALT was $86 \%$ in the Eastern cases and $72 \%$ in the Western cases. As for the topographic interrelation of both tumors, the majority of the cases $(54.7 \%)$ had independent tumors. Collision of both tumors was reported in 14 cases (26.4\%). There were 4 cases with contiguous and 5 cases with intermingling tumors.

Our case should be classified as intermingling collision tumor with $H$. pylori-negative DLBCL and adenocarcinoma in the stomach. Further, it should be noted that the gastric lymphoma in our case may not be primary but metastatic tumor from the cardiac legion since it was the largest (Fig. 1b). From this point of view, we considered the disease concept of "tumor-to-tumor metastasis, in which case the lymphoma cells had possibly metastasized into the gastric adenocarcinoma lesion, which was disrupted by rapidly growing lymphoma cells.

To the best of our knowledge, there has been no prior reported case of tumor-to-tumor metastasis of DLBCL to a primary gastric adenocarcinoma. Previously, one case of collision tumor of the stomach with both primary gastric lymphoma and adenocarcinoma was reported by 







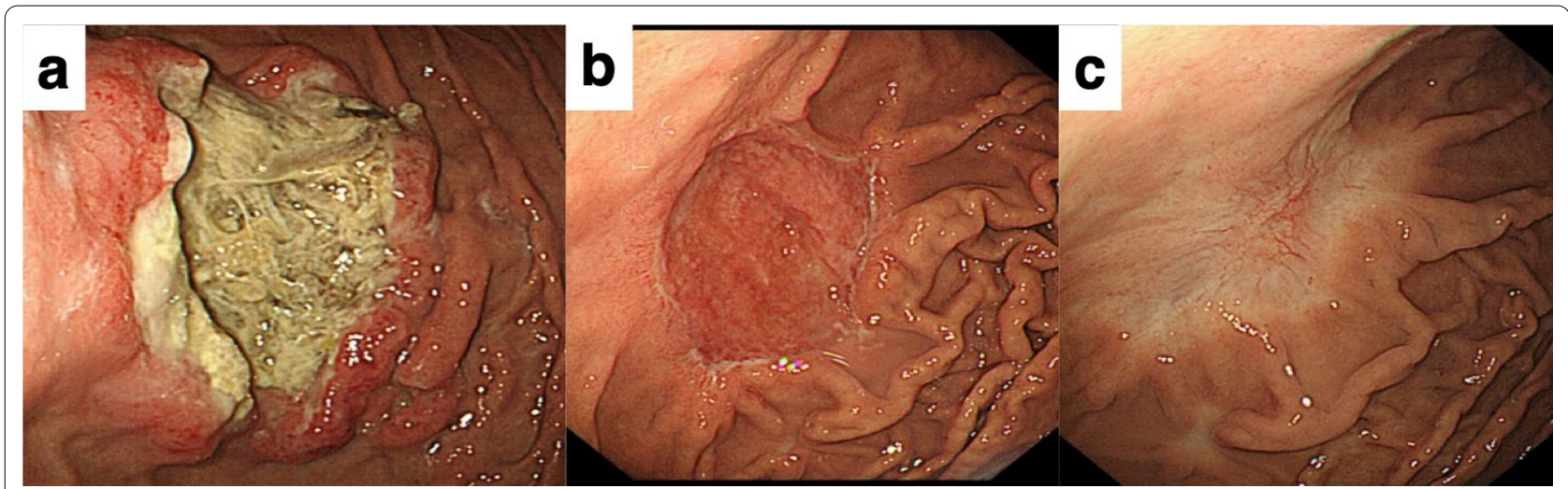

Fig. 3 Imaging findings. Follow-up EGD after chemotherapy detected a scar lesion instead of a tumor. a Before the first cycle of R-CHOP chemotherapy. b After the third cycle. c After the sixth cycle

Strofilas et al. [4]. They presented their case as tumorto-tumor metastasis since the pathological examination after the gastrojejunostomy showed the focal infiltration of adenocarcinoma into lymphomatous lymph node in the perigastric area. However, this is not a true definition of tumor-to-tumor metastasis according to the criteria given by Campbell Jr et al. [5]. Campbell excluded such cases from their review of tumor-to-tumor metastasis since the metastatic region is the lymphatic systems which were already the site of lymphatic malignancies. In addition, $\mathrm{Hu}$ et al. [6] reported a case of simultaneous occurrence of DLBCL at the ileocecal junction and gastric intramucosal adenocarcinoma; however, they did not metastasize into each other.

As for the mechanism by which one tumor metastasizes into another, it is reasonable to implicate the involvement of specific chemokines since a particular chemokine-receptor pair can serve as tissue-specific attractant molecules for tumor cells, promoting tumor cell migration to particular sites [7-9]. For example, a potential chemokine-receptor pair was examined by immunohistochemical staining in a patient with small cell lung carcinoma (SCLC) metastasizing to renal oncocytoma (RO) [10]. The SCLC cells were positive for CXCL12/SDF-1; however, both SCLC and RC cells were negative for CXCR4.

In this report, we successfully demonstrated the expression of both CXCL12/SDF-1 by the recipient adenocarcinoma cells and CXCR4 by the donor lymphoma cells. This is the first report which clearly suggests the possible involvement of CXCL12 (SDF-1)/CXCR4 axis in the process of tumor-to-tumor metastasis (Fig. 4). It is reported that $75.4 \%$ of DLBCL cells were positive for CXCR4 [11] and 77.3\% of gastric adenocarcinoma cells were positive for CXCL12/SDF-1 [12]. These previous findings would support the rationality of our results

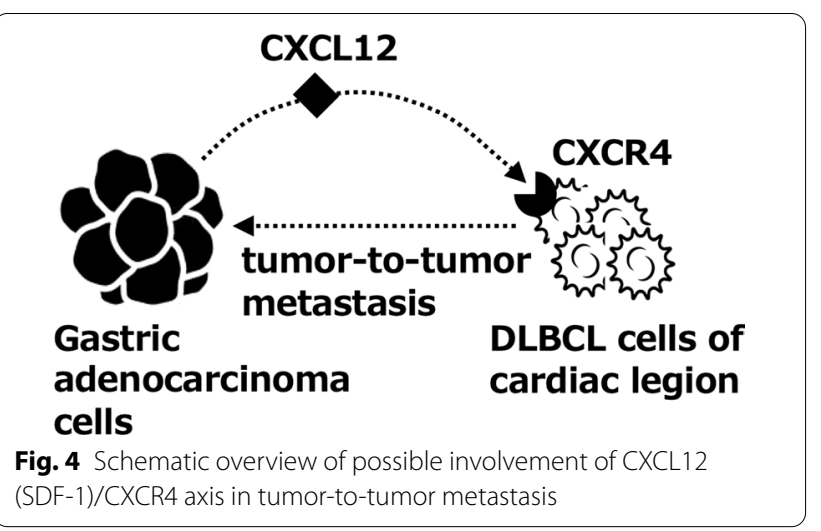

and conclusion. Additional evidence is needed in future detailed studies to evaluate the potential pivotal role of the CXCL12 (SDF-1)/CXCR4 axis in tumor-to-tumor metastasis, along with its previously described role in tumor development, survival, angiogenesis, metastasis, and tumor microenvironment.

In summary, our case suggests the involvement of CXCL12 (SDF-1)/CXCR4 axis in tumor-to-tumor metastasis of DLBCL to primary gastric adenocarcinoma.

\section{Abbreviations \\ DLBCL: Diffuse large B cell lymphoma; CXCL12: C-X-C motif chemokine ligand 12; SDF-1: Stromal cell-derived factor-1; CXCR4: C-X-C motif chemokine recep- tor 4; FDG: ${ }^{18}$ F-Fluorodeoxyglucose; EGD: Esophagogastroduodenoscopy; MALT: Mucosa associated lymphoid tissue; H. pylori: Helicobacter pylori; SCLC: Small cell lung carcinoma; RO: Renal oncocytoma; non-GCB: Non-germinal center B-cell-like.}

\section{Acknowledgements}

Not applicable.

\section{Authors' contributions}

YK performed the immunohistochemical staining. SM wrote the initial draft of the manuscript. SK provided critical analysis and review of the manuscript. AW 
and JM cared for the patient during his hospitalization. TS and NHD wrote the manuscript. All authors read and approved the final manuscript.

\section{Funding}

All authors declare that there is no funding to this publication.

\section{Availability of data and materials}

Not applicable.

\section{Declarations}

Ethics approval and consent to participate

Not applicable.

\section{Consent to publication}

Written informed consent was obtained from the patient for the publication of this Case report and any accompanying images.

\section{Competing interests}

The authors declare that they have no competing interests.

\section{Author details}

'Department of Hematology, Toyama University Hospital, 2630 Sugitani, Toyama 930-0194, Japan. ${ }^{2}$ Second Department of Internal Medicine, University of Toyama, Toyama, Japan. ${ }^{3}$ Division of Clinical Laboratory and Blood Center, Toyama University Hospital, Toyama, Japan. ${ }^{4}$ Division of Hematology/Oncology, University of Florida, Gainesville, FL, USA.

Received: 29 March 2021 Accepted: 14 June 2021

Published online: 29 June 2021

\section{References}

1. Takei H, Powell SZ. Tumor-to-tumor metastasis to the central nervous system. Neuropathology. 2009;29:303-8.
2. Petraki C, Vaslamatzis M, Argyrakos T, Petraki K, Strataki M, Alexopoulos C, et al. Tumor to tumor metastasis: Report of two cases and review of the literature. Int J Surg Pathol. 2003;11:127-35.

3. Hamaloglu E, Topaloglu S, Ozdemir A, Ozenc A. Synchronous and metachronous occurrence of gastric adenocarcinoma and gastric lymphoma: a review of the literature. World J Gastroenterol. 2006;12:3564-74

4. Strofilas A, Dalianoudis IG, Lagoudianakis EE, Genetzakis M, Tsekouras D, Chrysikos J, et al. Collision tumour of the stomach with a cancer to cancer metastasis: a case report. Cases J. 2008;1:63.

5. Campbell LV, Gilbert E, Chamberlain CR, Watne AL. Metastases of cancer to cancer. Cancer. 1968;22:635-43.

6. Hu XR, Hu YX, Fu HR, Sheng LX, Huang WJ, Hu KY, et al. Diffuse large B-cell lymphoma with concurrent gastric adenocarcinoma: Case report and literature review. J Int Med Res. 2011;39:2051-8.

7. Tanaka T, Bai Z, Srinoulprasert Y, Yang BG, Hayasaka H, Miyasaka $M$. Chemokines in tumor progression and metastasis. Cancer Sci. 2005;96:317-22.

8. Mortezaee K CXCL12/CXCR4 axis in the microenvironment of solid tumors: a critical mediator of metastasis. Life Sci. 2020;249:117534.

9. Britton C, Poznansky MC, Reeves P. Polyfunctionality of the CXCR4/ CXCL12 axis in health and disease: implications for therapeutic interventions in cancer and immune-mediated diseases. FASEB J. 2021;35:e21260.

10. Peguero JSN, Camacho LH, Kemp B, Campos LT. Chemokine expression in tumor-to-tumor metastasis. Oncol Lett. 2010;1:449-52.

11. Du H, Zhang L, Li G, Liu W, Tang W, Zhang H, et al. CXCR4 and CCR7 expression in primary nodal diffuse large B-cell lymphoma-a clinical and immunohistochemical study. Am J Med Sci. 2019;357:302-10.

12. Zheng S, Shi L, Zhang Y, He T. Expression of SNCG, MAP2, SDF-1 and CXCR4 in gastric adenocarcinoma and their clinical significance. Int J Clin Exp Pathol. 2014;7:6606-15.

\section{Publisher's Note}

Springer Nature remains neutral with regard to jurisdictional claims in published maps and institutional affiliations.
Ready to submit your research? Choose BMC and benefit from:

- fast, convenient online submission

- thorough peer review by experienced researchers in your field

- rapid publication on acceptance

- support for research data, including large and complex data types

- gold Open Access which fosters wider collaboration and increased citations

- maximum visibility for your research: over $100 \mathrm{M}$ website views per year

At BMC, research is always in progress.

Learn more biomedcentral.com/submissions 